\title{
Microbiology Specimen Link Group Identifier
}

National Cancer Institute

\section{Source}

National Cancer Institute. Microbiology Specimen Link Group Identifier. NCI Thesaurus.

Code C162028.

A sequence of characters used to link multiple microbiology specimen records to a single finding. 